https://doi.org/10.48009/2_iis_2009_249-256

\title{
GLOBALIZATION, COLLABORATION, AND SOCIAL NETWORKING: AN EXPLORATORY STUDY
}

\author{
Robert L. Totterdale, Robert Morris University, rtotterdale@tbconsulting.biz
}

\begin{abstract}
Individuals utilize a variety of tools to collaborate and communicate as part of a global organization. Social networking tools such as wikis, blogs, and MySpace are increasingly being used both on a personal basis and within corporations, while collaboration tools such as Microsoft's Sharepoint services are being used to meet corporate information needs. With the expanding adoption of these tools, this study investigates their usage by conducting a survey of 785 individuals in a professional services firm. In addition to understanding the frequency of use of the various tools, this study evaluates if the usage is influenced by age, gender, or geographic operating location (i.e. U.S., India, China, and Europe).
\end{abstract}

Keywords: Globalization, Collaboration, Social Networking, Information Technology, Sharepoint

\section{INTRODUCTION}

To operate effectively, global organizations must have in place technologies and business processes that support communications and collaboration both internally and with their business partners. However, even with these systems in place, factors such as geographic dispersion of personnel, technology, culture, workforce composition, and regulatory requirements can impact how well individuals can connect with one another or in groups.

Numerous tools and technologies have been deployed in global workforces to unite workgroups and individuals to complete their work more efficiently. Tools such as Microsoft's Sharepoint Services 2007, Microsoft's Outlook, IBM Lotus Notes, and others have been used to support document and email management and to enhance communications both within and between businesses.
As businesses have continued to implement and utilize various collaboration technologies, social networking and Web 2.0 technologies have enjoyed considerable success in the consumer marketplace, and increasingly are being adopted by corporations to expand their communications and collaboration capabilities. Capabilities such as wikis, blogs and RSS feeds have been integrated with more traditional collaboration products such as Microsoft's Sharepoint 2007 to deliver more functionality to users who are seeing the benefit of using these tools to support business operations.

This study explores the use of collaboration and social networking tools by professionals in a global technology firm with over 100,000 employees. Using survey responses from 785 professionals and executive interviews, relationships between age, gender, and geography (i.e. home country), and the usage of social networking tools were investigated. In contrast to other research that has been performed, this study assesses whether professionals that use traditional collaboration tools are also more likely to use social networking tools.

Four research questions addressed by this study include:

R1. What major collaboration tools are used by the global organization and what is the frequency of their use?

R2. How often are wikis, blogs and MySpace used by business professionals?

R3. Do relationships exist between age, gender, country and the use of social networking tools?

R4. How does the use of Sharepoint relate to the use of social networking tools?

\section{LITERATURE REVIEW}


Since this study is focused on the usage of social networking and collaboration tools both from a corporate and personal perspective, this section provides definitions of key terms, and highlights findings relating to usage in organizations as well as broader use of the technologies. Each is described below.

Multiple and varied definitions exist to describe "people to people" communications and collaboration using Web based technologies. Some suggest the term social networking, while others use the term Web 2.0, which is often used in the context of consumer interactions [4]. In addition, Enterprise 2.0 is another term offered by AIIM [9], the Enterprise Content Management Association that defines it as "a system of Web-based technologies that provide rapid and agile collaboration, information sharing, emergence, and integration capabilities in the extended enterprise" (p. 11). This definition is related to the application of the social networking technologies in a business environment.

Regardless of the definition that is used, wikis and blogs are typically cited as examples of technologies that are used to facilitate communications and collaboration either in a corporate or personal environment $[13,8]$. Hasan and Pfaff [13] define a wiki as "a web-based application that allows many participants to write collaboratively, where they can continue to add or edit the content of documents and dynamically determine relationships between sets of documents" (p. 41). In contrast, a blog is defined by Agarwal and Liu [1] as "a website that displays, in reverse chronological order, the entries by one or more individuals and usually has links to comments on specific postings" ( $p$. 18). Both wikis and blogs are used in businesses and are also widely used in the consumer marketplace $[1,8,12]$. MySpace is another social networking technology that has been used extensively by individuals for sharing both personal and business information [16]. It has also been used by corporations to conduct research as part of the hiring process [11]. Wikis, blogs, and MySpace have all been deployed on a global basis with millions of users $[2,14,15$, 16].

In corporations, research conducted by AIIM found that a majority of users agreed that wikis, blogs, other social networking tools were key components of an Enterprise 2.0 platform [9].
Additionally, they found that the impact of these tools in the organization should be to increase collaboration and to increase the capture of corporate knowledge; both of which are key components of knowledge management in an organization. Another finding in the AIIM research was that culture was a significant determinant of the adoption of the technology in an organization.

Further research cited by Brodkin [6] found that there were no significant differences in attitudes toward Web 2.0 technologies between different age groups. Other findings by APQC [8] in case studies conducted at Royal Dutch Shell PLC, Siemens, and the U.S. Department of State, indicated that various technologies such as Sharepoint, wikis, blogs, interactive messaging, and Email have been successfully deployed and are components of an overall collaboration and knowledge management strategy. For the companies in the case studies, the use of wikis and blogs has not been mandated within the organizations. In one of the cases that considered tool usage over a four week period, $77 \%$ of the users posted responses and $19 \%$ created blog posts indicating a high level of usage.

Other research from Gareiss [10] suggests that wiki use is higher for companies that have teleworking policies ( $57 \%$ versus $44 \%$ for those without a policy) and that blog usage is higher as well $(41 \%$ versus $39 \%$ for those without a policy). Gibson [12] argues that the growth of wikis and blogs has been proliferating virally as he cites companies such as Motorola that has over 1000 wikis, 2600 blogs, and over 17 terabytes of searchable data. Others such as Brooks [7] indicate that the "biggest blogging community is now in China" (p. 19), a reflection of the growth of this technology around the world. Similarly, Liu [2] suggests that blogs are becoming a major marketing tool for enterprises in China. Lastly, a Snyovate Marketing Survey [3] found that awareness and usage of blogs strongly correlates with age, that women are more likely than men to blog, and younger people are more active bloggers.

Recent research by the PEW Internet \& American Life Project [16] has found that one third $(35 \%)$ of American adult internet users has a profile on a social networking site such as MySpace. Further, the findings show that younger adults have higher usage rates than older adults. Additionally, this research found that men 
and women are equally likely to visit a social networking site, and that less than a third of social network users were found to use them for professional purposes. In another study, Majchrzak, Wagner, and Yates [18] found that wiki use in a corporation is sustainable and that benefits relating to enhanced reputation, making work easier, and improved business processes were achievable. This finding lends support for the expansion of wikis in the enterprise.

\section{RESEARCH METHODOLOGY}

This report was based on survey data obtained from a global professional services firm during a two week period in October and November of 2008. The objective of the study was to investigate the effects of age, gender, and geography on the use of collaboration and social networking tools. In addition, the research was also intended to determine if relationships existed between the use of a specific collaboration tool, Sharepoint, and social networking tools such as wikis, blogs, and Myspace.

An established research strategy, surveys were used for data collection [5, 19]. Given the size of the organization participating in the research, and the need to capture information both quickly and economically, the survey provided a mechanism for identifying the attributes of a large population from a smaller group [5]. The survey was cross sectional where the data was collected at one point in time and was administered on-line through Zoomerang, an internet survey tool.

The professional services organization had over 100,000 employees at the time the research was conducted. Executives from the firm agreed to have six of their project teams or groups made up of 1,985 personnel participate in the study. This convenience sample, which included multiple age groups and geographies, was considered to be representative of the broader organization. Since the email addresses of the 1,985 participants were known, a single stage sampling procedure was used and $100 \%$ of the population was sent the link to the electronic survey instrument. The survey tool captured 785 responses which reflected a $40 \%$ response rate for the research.

The survey instrument was constructed in a three step process. In the first step, interviews were conducted with senior executives to gather information about the different technologies used to support collaboration in the organization and to gain an understanding of terminology used to describe the technology internally. From the interviews, the primary technologies identified were Sharepoint, Outlook, Interactive Messaging (IM), Lotus Notes, ClearCase, and shared folders. Although other technologies were in use, these were believed to be the most frequently used and were therefore included in the survey in questions relating to tool usage. With respect to social networking technologies, the researcher elected to include survey options of wikis, blogs, and MySpace since they have been acknowledged by others as being widely used in the consumer marketplace $[8,9,16]$.

After defining the collaboration tools in use, the second step in the development of the survey instrument was to define the response categories or scales to gather usage information from the participants. For tool usage, a five point Likerlike scale consisting of "Not at All," "Seldom," "Occasionally," "Often and Frequently" was used to collect frequency information. Age groupings used in the survey included $<26,26$ $30,31-36,37-47$, and 48 or more. Regarding the volume of email participants received on a daily basis, averages of <20, 21-50, 51-100, 101-200, and over 200 were listed as possible responses.

From a geographic perspective, the survey provided response options of U.S., China, Germany, India, Spain and Other. For the "Other" category, participants could write in a country if it were not listed. Since there were a small number of responses for the European countries, all continental Europe responses were recoded as Europe for analysis purposes. All other responses in the "Other" category were eliminated from the analysis because of the small number of respondents.

The last step in preparing the instrument was to test it with a group of seven individuals to insure that the questions were understood and that the electronic data collection worked properly. After making minor wording changes to the instrument as a result of participant feedback, the results were downloaded from Zoomerang and uploaded into SPSS to validate the data transfer.

After the survey instrument was finalized, a senior executive from each of the groups sent a request to all of their team members inviting 
them to participate in the research. A brief introduction to the research indicated that their involvement was voluntary. To improve the response rates to the survey, each executive also sent a follow-up email encouraging participation approximately one week after the initial survey invitation was sent.

Four independent variables (age, gender, geography, and Sharepoint use) were evaluated separately against the dependent variables (collaboration technologies and social networking technologies) in the analysis. Recoding of the variables was performed as shown in Figure 1 to facilitate the analysis of the data.

\begin{tabular}{|l|l|l|}
\hline Variable & $\begin{array}{l}\text { Original } \\
\text { Response }\end{array}$ & \multicolumn{1}{|c|}{$\begin{array}{c}\text { Recoded } \\
\text { Response }\end{array}$} \\
\hline Age & $\begin{array}{l}37-47 \\
48 \text { or } \\
\text { older }\end{array}$ & $\begin{array}{l}\text { Over 37 } \\
\text { Over 37 }\end{array}$ \\
\hline $\begin{array}{l}\text { Geograp } \\
\text { hy }\end{array}$ & $\begin{array}{l}\text { Spain } \\
\text { Germany } \\
\text { Other } \\
\text { (continent } \\
\text { al }\end{array}$ & $\begin{array}{l}\text { Europe } \\
\text { Europe } \\
\text { Europe } \\
\text { Excluded/miss } \\
\text { ing data }\end{array}$ \\
& $\begin{array}{l}\text { Other } \\
\text { (not }\end{array}$ & \\
& Europe) & \\
\hline Usage & $\begin{array}{l}\text { Seldom } \\
\text { Often }\end{array}$ & $\begin{array}{l}\text { Occasionally } \\
\text { Frequently }\end{array}$ \\
\hline Email & $<20$ and & Low/medium \\
Usage & $21-50$ & volume \\
& $51-100$ & Heavy volume \\
& $101-200$ & Heavy volume \\
& Over 200 & Heavy volume \\
\hline
\end{tabular}

Figure 1 Recoding of Variables

Upon closing the survey process, survey feedback was downloaded from Zoomerang and uploaded into SPSS for reporting and analysis. SPSS was used to generate frequency distributions for the survey responses, and was also used to perform chi-square calculations to determine if statistical relationships existed between tool usage and key demographic variables.

\section{Results}

Results from this study reflect qualitative information obtained from interviews regarding current technologies in place in the organization and quantitative results obtained from the electronic survey. Each of these is described more fully in the following sections.

Interviews of executives from the organization identified a number of tools and technologies that were in use in the company and which were being made available to the professional services organization. An executive from the CIO's organization indicated over 15,000 instances of Sharepoint were in use in the organization and was the default technology often selected by project teams when client requirements did not dictate otherwise. In addition to Sharepoint, the executive indicated that Lotus Notes, Shared Folders, interactive messaging, and Microsoft Outlook for email were frequently used on a global basis to support communications, collaboration, and knowledge management. Other executives participating in the interviews also cited ClearCase and ClearQuest, document management tools from IBM, as other tools that were being used.

In addition to the broadly deployed collaboration tools listed above, the executive also indicated that newer technologies were being made available to professionals on a global basis. Examples of these technologies were videoconferencing, wikis, blogs, and a video sharing tool comparable to You Tube. These technologies were newer to the organization, were not as widely deployed as Sharepoint, and although made available to the organization, their use was not mandated. Further, social networking applications such as people profiles and social bookmarking were also mentioned as emerging uses of existing technology. As an example of how the social networking technology has been adopted in the organization, an executive cited a situation "where a blog was set-up and without any organizational notification of its existence, close to 3,000 blog posts occurred over a relatively short period of time."

For each of the research questions, a summary of the key findings are summarized below. The first research question (R1) investigated the type and frequency of use for the major collaboration tools in the organization. The results from the survey, as shown in Table 1, indicated that Sharepoint, shared folders, and interactive messaging (IM) were the three most frequently utilized collaboration tools (i.e. they had the 
lowest number of respondents indicating that they did not use the tool at all). Usage of another collaboration tool, Microsoft Outlook was assessed by examining the volume of email processed by individuals. Findings indicated that $66 \%$ of the respondents received less than 50 emails a day while $34 \%$ indicated that they received between 51 to over 200 emails per day.

\begin{tabular}{|lc|ccc|c|}
\hline Collaboration Tools - \% Usage & & Frequently & Occasionally & Not At All & Freq+Occ \\
\hline Sharepoint & $\mathrm{n}=785$ & 37 & 45 & $\mathbf{1 8}$ & $\mathbf{8 2}$ \\
Lotus Notes & $\mathrm{n}=785$ & 38 & 12 & $\mathbf{5 0}$ & $\mathbf{5 0}$ \\
ClearCase & $\mathrm{n}=785$ & 12 & 18 & $\mathbf{7 0}$ & $\mathbf{3 0}$ \\
Shared Folders & $\mathrm{n}=785$ & 57 & 26 & $\mathbf{1 7}$ & $\mathbf{8 3}$ \\
Interactive Messaging (IM) & $\mathrm{n}=785$ & 74 & 17 & $\mathbf{9}$ & $\mathbf{9 1}$ \\
\hline
\end{tabular}

Table 1 Collaboration Tool Usage

In addition to determining the usage of internal collaboration tools in $\mathrm{R} 1$, the second research question (R2) investigated the usage of social networking tools by professionals in the organization. Individuals using these tools could have used them personally or within the organization. Findings from the survey are summarized in Table 2. Of the three social networking tools, wikis were used by $41 \%$ of the respondents while blogs and MySpace were used by $39 \%$ and $25 \%$ of the respondents respectively.

\begin{tabular}{|c|c|c|c|c|c|}
\hline \multicolumn{2}{|c|}{ Social Networking Tools -\% Usage } & Frequently & Occasionally & Not At All & Freq $+\mathrm{Occ}$ \\
\hline Wikis & $n=785$ & 14 & 27 & 59 & 41 \\
\hline Blogs & $n=785$ & 9 & 30 & 61 & 39 \\
\hline Myspace & $n=785$ & 5 & 20 & 75 & 25 \\
\hline
\end{tabular}

Table 2 Social Networking Tool Usage

Once the usage of the collaboration tools and social networking tools was determined, the next process was to evaluate whether there were any statistically relevant relationships between the independent variables age, gender, geography, and Sharepoint usage with the dependent variables wikis, blogs, and MySpace. With respect to gender and the use of social networking tools, no statistical relevance was suggested by the data. In contrast, a relationship did exist between geography and the usage of MySpace, but not for the other tools. Although not at a statistically relevant level, the Chinese did indicate a much higher level usage of blogs than the other countries in the analysis. A summary of the geographic statistics are listed in Table 3.

\begin{tabular}{|cccccccccc|}
\hline Geography $\mathbf{n = 7 7 2}$ & $\begin{array}{c}\text { Basis } \\
\text { \%Usage }\end{array}$ & U.S. & China & Europe & India & $\underline{\boldsymbol{x}}_{-}^{2}$ & $\underline{\text { df }}$ & $\underline{\mathbf{p}}$ \\
\hline Wikis & Not At All & 60 & 68 & 54 & 58 & 10.088 & 6 & 0.121 \\
Blogs & Not At All & 61 & 44 & 70 & 61 & 9.047 & 6 & 0.171 \\
Myspace & Not At All & 77 & 38 & 87 & 75 & 29.388 & 6 & $\mathbf{0 . 0 0 1}$ \\
Sharepoint & Not At All & 17 & 24 & 8 & 22 & 19.696 & 6 & 0.003 \\
\hline
\end{tabular}

Table 3 Geography and Social Networking Relationships

With respect to the influence of age on the use of social networking tools (R3), a statistically significant relationship was found that indicated that younger respondents were more likely to use a wiki than older respondents. In addition, the relationship between age and the use of MySpace 
was found to be approaching a level of statistical significance. Based on percentages of participant responses indicating usage of MySpace, younger respondents were more likely than older respondents to use the tool. A summary of key findings relating to age are shown in Table 4 .

\begin{tabular}{|ccccccccc|}
\hline Age N=785 & $\begin{array}{c}\text { Basis } \\
\text { \%Usage }\end{array}$ & $\begin{array}{c}\text { Years } \\
<\mathbf{2 6}\end{array}$ & $\mathbf{2 6 - 3 0}$ & $\mathbf{3 1 - 3 6}$ & Over $\mathbf{3 7}$ & $\underline{\mathbf{x}}_{-}^{\mathbf{2}}$ & $\underline{\mathbf{d f}}$ & $\underline{\mathbf{p}}$ \\
\hline & & & & & & & & \\
Wikis & Not At All & 44 & 58 & 64 & 72 & 39.765 & 6 & $\mathbf{0 . 0 0 1}$ \\
Blog & Not At All & 55 & 60 & 65 & 64 & 6.254 & 6 & 0.395 \\
Myspace & Not At All & 71 & 72 & 79 & 82 & 11.773 & 6 & $\mathbf{0 . 0 6 7}$ \\
Sharepoint & Not At All & 20 & 18 & 16 & 17 & 2.463 & 6 & 0.872 \\
& & & & & & & & \\
\hline
\end{tabular}

Table 4 Age and Social Networking Relationships

A final area of investigation was to determine if any relationships existed between the use of Sharepoint, a primary collaboration tool in the organization, and the use of the social networking tools (R4). For all three of the social networking tools identified in the survey, a statistically relevant relationship was found between the usage of the social networking tool and the usage of Sharepoint. In order words, the analysis showed that professionals who used Sharepoint were more likely to also use wikis, blogs, and MySpace than those who did not use Sharepoint. A summary of the results are provided in Table 5.

\begin{tabular}{|c|c|c|c|c|}
\hline Sharepoint & $\mathrm{n}=785$ & $\underline{x}^{2}$ & $\underline{\mathrm{df}}$ & $\underline{p}$ \\
\hline Wikis & & 13.851 & 2 & 0.001 \\
\hline Blogs & & 14.428 & 2 & 0.001 \\
\hline MySpace & & 14.53 & 2 & 0.001 \\
\hline
\end{tabular}

Table 5 Sharepoint -Social Networking Relationships

\section{CONCLUSIONS}

Through a survey of 785 professionals in a global organization, this research study has identified the frequency of use of certain collaboration and social networking tools in the company. Further, the study has found that significant relationships exist between the usage of these tools, certain age groups, and geographic operating locations. However, no relationships were found with respect to gender and the use of the various tools. In addition, the findings indicated that users of the Sharepoint collaboration tool were more likely than non
Sharepoint users to utilize the various social networking tools.

Prior research from Gareiss [10], which indicated that in teleworking companies, $44 \%$ of the employees used wikis and $39 \%$ used blogs, was confirmed with this studies findings of $41 \%$ wiki use and 39\% blog use. Another finding from the Snyovate study [3] that indicated a strong correlation between age and the use of blogs could not be confirmed statistically. However, a higher percentage of younger participants in the study did indicate a higher level of blog usage than the older groups. In addition, the research confirmed Lenhart's [16] findings that suggested that men and women were equally likely to use social networking tools. Similar to Lenhart's findings, this study also confirmed that younger individuals were more likely than older individuals to use social networking tools; although only wikis usage was found to be at a statistically significant level. Lastly, China demonstrated the highest level of blog usage of all of the geographies, a finding which was consistent with arguments made by Brooks [7] regarding the exploding growth of internet users in that country.

The findings of the study can provide valuable insights to organizations considering the adoption of social networking tools. Given differences in usage by age groups and geographies, this information can be used to assist in determining underlying causes for lower levels of adoption, and to address these issues if possible. The demonstrated relationship between existing collaboration tool users and social networking tools could also be explored for opportunities to improve adoption. 
Although a large number of participants completed the survey, several limitations involving this research must be considered. Because the research was conducted at a single company, it is not possible to generalize the results to a larger population. In addition, the relatively smaller number of participants from Europe and China may limit the effectiveness of the analysis for these countries. Lastly, the scope of the analysis was to investigate the use of wikis, blogs and MySpace without considering whether they were used professionally or on a personal basis. This distinction may be important to understand differences in adoption rates.

With these limitations, opportunities exist for further research. Expanding the number of companies and countries in the analysis would help to confirm the findings from this study and to gain new insights. Additionally, exploring the impact of security/privacy concerns and legal systems on an individual's usage may be helpful in interpreting usage differences between groups. Lastly, research directed at gaining a more detailed understanding of how social networking tools are used to support business and personal needs can be used to develop new approaches for improving the adoption of the technology.

\section{References}

1. Agarwal, N., \& Liu, H. (2008). Blogosphere: Research Issues, Tools and Applications. ACM-SIGKDD Explorations, 10(1).

2. Anonymous. (2007a). CCID Consulting's Internet Industry Research Center Reviews China's Web2.0 in 2006 and Forecasts Developments in 2007. PR Newswire.

3. Anonymous. (2007b). Why do you read a blog? Survey tracks the blogging behavior of Americans. Public Relations Tactics, 14(11), 21.

4. Anonymous (2008). Web 2.0: Building the Nex-Generation Workplace- the Driving Force Behind Just about Every Aspect of the Enterprise Web 2.0, is of Course, the User.
5. Babbie, E. (2007). The practice of social research (11th ed.). Belmont, CA: Thomson.

6. Brodkin, J. (2008). Enterprise 2.0 generational divide largely a myth, study finds. Network World (Online).

7. Brooks, G. (2009). BLOGGING: Natural selection. New Media Age, 19.

8. Carlin, S., Lee, J., Lemons, D., O'Dell, C., \& Swift, G. (2008). The Role of Evolving Technologies: Accelerating Collaboration and Knowledge Transfer: APQC.

9. Frappaolo, C., \& Keldsen, D. (2008). Enterprise 2.0: Agile, Emergent \& Integrated: AIIM.

10. Gareiss, R. (2008). Teleworking drives Web 2.0 applications. Network World (Online).

11. Genova, G. (2009). No Place to Play: Current Employee Privacy Rights in Social .Networking Sites. Business Communication Quarterly, March.

12. Gibson, S. (2006). Veni, Vidi, Wiki; Wikis are spreading virally, especially in recent months, as their benefits become widely recognized. eWeek, 23(46), 22.

13. Hasan, H., \& Pfaff, C. (2006). The Wiki: a tool to support the activities of the knowledge worker. Paper presented at the Proceedings at the Transformational Tools for 21st Century

14. Hsu, C.-L., \& Lin, J. C.-C. (2008). Acceptance of blog usage: The roles of technology acceptance, social influence and knowledge sharing motivation. Information \& Management, 45(1), 65.15. Kharif, O., \& Lakshman, N. (2007). MySpace, Facebook Get Serious About Asia. Business Week (Online), 1.

16. Lenhart, A. (2009). Pew Internet Project Data Memo. Washington, DC: Pew Internet \& American Life Project. 
17. Lu, H.-P., \& Hsiao, K.-L. (2007). Understanding intention to continuously share information on weblogs. Internet Research, 17(4), 345.

18. Majchrzak, A., Wagner, C., \& Yates, D. (2006). Corporate wiki users: results of a survey. Paper presented at the Proceedings of the 2006 international symposium on Wikis.

19. Pattern, M. L. (2001). Questionnaire Research - A Practical Guide (Second ed.). Los Angeles, CA: Pyrczak Publishing. 